Chapman University

Chapman University Digital Commons

Pharmacy Faculty Articles and Research

School of Pharmacy

2012

\title{
Confounding Issues in Estimation of Patient- Specific Pharmacokinetic Parameters and Dosage Individualization of Aminoglycosides
}

Reza Mehvar

Chapman University, mehvar@chapman.edu

Follow this and additional works at: http://digitalcommons.chapman.edu/pharmacy_articles

Part of the Other Pharmacy and Pharmaceutical Sciences Commons, Pharmaceutical Preparations Commons, and the Pharmaceutics and Drug Design Commons

\section{Recommended Citation}

Mehvar R, Confounding Issues in Estimation of Patient-Specific Pharmacokinetic Parameters and Dosage Individualization of Aminoglycosides. Curr. Clin. Pharmacol., 7:28-35 (2012). doi: 10.2174/157488412799218833

This Article is brought to you for free and open access by the School of Pharmacy at Chapman University Digital Commons. It has been accepted for inclusion in Pharmacy Faculty Articles and Research by an authorized administrator of Chapman University Digital Commons. For more information, please contact laughtin@chapman.edu. 


\section{Confounding Issues in Estimation of Patient-Specific Pharmacokinetic Parameters and Dosage Individualization of Aminoglycosides}

\section{Comments}

This is a pre-copy-editing, author-produced PDF of an article accepted for publication in Current Clinical Pharmacology, volume 7, in 2012 following peer review. The definitive publisher-authenticated version is available online at DOI: 10.2174/157488412799218833.

\section{Copyright}

Bentham Science Publishers 


\section{Confounding Issues in Estimation of Patient-Specific Pharmacokinetic Parameters and Dosage Individualization of Aminoglycosides}

Reza Mehvar*

Department of Pharmaceutical Sciences, School of Pharmacy, Texas Tech University Health

Sciences Center, Amarillo, Texas 79106, USA

Correspondence to: Reza Mehvar, Pharm.D., Ph.D., School of Pharmacy, Texas Tech University Health Sciences Center, 1300 S. Coulter, Amarillo, TX 79106, USA, Telephone: (806) 356-4015 Ext.337, FAX: (806) 356-4034. E-Mail: reza.mehvar@ttuhsc.edu

Running Title: Confounding Issues in Aminoglycoside Dosing 
Abstract: Aminoglycoside antibiotics are usually administered by multiple short intravenous infusions at fixed intervals. Today, equations reported 35 years ago by Sawchuk and Zaske are still the cornerstone of methods used for determination of patient-specific pharmacokinetic parameters of aminoglycosides and individualization of drug dosage regimens in many clinical settings. Additionally, these methods are included in many clinical pharmacology curricula in pharmacy and other related fields. However, there are a few issues with regard to the application and/or modification of this method in clinical settings, which may result in some confusion among novice clinicians. For example, serum samples collected from different intervals at steady state, instead of samples obtained during the same interval, require special manipulation of sampling time before they can be used for estimation of pharmacokinetic parameters. Furthermore, there are various ways that the original equations are modified or simplified, which can result in some degree of error in the estimates of pharmacokinetic parameters and ensuing dosage regimen calculations. Simulation data presented here indicate that in some cases, these errors may be substantial, depending on the length of short infusion, half life of the drug, and the dosage interval. For instance, using equations developed for intravenous bolus mode of administration, ignoring the short infusion, may result in $\geq 25 \%$ error for a typical patient and dosing scenario. Although experts may use modified equations, understanding their error ramifications, these modifications may be confusing to the novice clinicians. Therefore, it is recommended that exact equations developed specifically for multiple intravenous infusions be used without any modification, particularly in settings where clinicians are being trained.

Key Words: Aminoglycoside dosing, clearance, clinical pharmacokinetics, elimination rate constant, intermittent intravenous infusion, patient-specific pharmacokinetics, peak and trough, volume of distribution. 


\section{INTRODUCTION}

Aminoglycosides are bactericidal antibiotics, used systemically in the treatment of serious gram negative infections, such as pneumonia or bacteremia [1]. The most widely used systemic aminoglycosides include amikacin, gentamicin, tobramycin, and netilmicin. The efficacy and toxicity of these drugs are related to their plasma concentrations [2, 3]. Additionally, the pharmacokinetics of aminoglycosides are subject to substantial intra- and interpatient variability $[4,5]$. Therefore, their dosing in most cases is guided by therapeutic drug monitoring. The absorption of aminoglycosides after oral administration is generally poor. Therefore, for systemic effects, they are administered mostly by intramuscular or intravenous injections. However, the intermittent intravenous (iv) infusion of these drugs over 30-60 min at fixed intervals is by far the most common method used for their administration.

The intermittent iv infusion method is a unique method of drug administration, which is different from both constant iv infusion and iv bolus injection. Therefore, specific equations are used for calculation of some of the kinetic parameters after this mode of administration. Thirty five years ago [6], Drs. Ronald Sawchuk and Darwin Zaske, from the University of Minnesota College of Pharmacy, reported a general method for estimation of the patient-specific kinetic parameters of aminoglycosides and the design of dosage regimens. To date, this method is still the cornerstone of aminoglycoside dosage individualization in most clinics, although extended interval aminoglycoside dosing was also added later to the methods of aminoglycoside administration [7]. Therefore, the so-called Sawchuk-Zaske method is one of the major methods used by clinicians to initiate and/or adjust aminoglycoside dosing. Additionally, it is the main method that is taught in pharmacy and medicine curricula. 
Despite its wide acceptance, there are some issues with regard to the application and/or modification of this method in clinical settings, which may result in some confusion and errors in estimation of pharmacokinetic parameters and any patient-specific dosage regimens designed from the calculations. Although most of these issues may be viewed as minor nuisances for expert clinicians, they may pose significant sources of confusion for future clinicians during their early stages of understanding these methods. The purpose of the current communication is, therefore, to address these confounding issues and their potential impact on the estimation of the patient-specific pharmacokinetic parameters and/or dosing of patients, in a style that may be used for education of novice clinicians. Where modifications of the original equations are discussed, simulations are used to provide data on the degree of error associated with these modifications. This communication is not intended to be an exhaustive review of the aminoglycoside pharmacokinetics, pharmacodynamics, population-based initial dosing, or extended-interval dosing. For the latter purposes, the reader is referred to excellent book chapters [8-10] and review articles [11-13] on these topics, which were published recently.

\section{HOW IS INTERMITTENT INTRAVENOUS INFUSION DIFFERENT FROM INTRAVENOUS BOLUS ADMINISTRATION?}

When a drug is administered by the iv bolus method, the entire dose is introduced into the systemic circulation relatively rapidly (within a few seconds to minutes), resulting in a maximum plasma concentration $\left(\mathrm{C}_{\max }\right)$ being achieved almost immediately $\left(\mathrm{C}_{\mathrm{o}}\right)$. With a short iv infusion, however, each dose is administered over a short, constant rate infusion, resulting in a lower $\mathrm{C}_{\max }$, compared with the administration of the same dose of the drug by the iv bolus method (Fig. 1). However, as demonstrated in Fig. 1, the minimum plasma concentration before the 
administration of the next dose $\left(\mathrm{C}_{\min }\right)$ using the short infusion method is expected to be higher than that after the iv bolus dose. Overall, a lower $\mathrm{C}_{\max }$ and higher $\mathrm{C}_{\min }$ mean a lower fluctuation in the plasma concentrations after intermittent short iv infusion, compared with multiple bolus doses. Obviously, with linear pharmacokinetics (i.e. where clearance and volume of distribution remain the same with different plasma concentrations), total exposure (AUC) for both cases will be the same. Although more inconvenient than iv bolus dose, this method has the advantage of avoiding high initial concentrations which may produce toxicity. An additional reason for using this method for aminoglycosides is to allow time for distribution of the drug into the tissues, allowing the use of simple one-compartment model kinetics.

\section{ESTIMATION OF PATIENT-SPECIFIC PHARMACOKINETIC PARAMETERS}

The normal dosing of aminoglycosides is via administration of multiple short $(0.5-1 \mathrm{~h})$ iv infusions of the drug at fixed intervals $(\tau)$. This results in a $\mathrm{C}_{\max }$, attained at the end of the short infusion and a $\mathrm{C}_{\min }$, attained at the end of dosage interval before the next dose is administered (Fig. 1). Although several samples, belonging to the same or multiple intervals, may be taken from a patient and subjected to pharmacokinetic software for analysis, a common method is obtaining the kinetic parameters by subjecting the data from a peak and a trough sample to the Sawchuk-Zaske method (Fig. 1). The peak concentration is measured in a sample taken some time (normally at least $30 \mathrm{~min}$ after a $0.5 \mathrm{~h}$ infusion or $15-30 \mathrm{~min}$ after a $1 \mathrm{~h}$ infusion) after the infusion is stopped. Regardless of the length of infusion, it is recommended that the peak sample be taken at $\geq 1 \mathrm{~h}$ after the start of infusion to avoid the distribution phase. A trough concentration is normally taken $\leq 30 \mathrm{~min}$ before the next dose is administered (e.g., $30 \mathrm{~min}, 15 \mathrm{~min}$, or 
immediately before the next dose). In the following sections, the use of peak and trough samples in obtaining patient-specific kinetic parameters is explained.

\section{Estimation of Elimination Rate Constant: Peak and Trough from the Same or Different Dosage Intervals?}

Although in their original study [6], Sawchuk and Zaske obtained four plasma samples from each patient at steady state (one predose sample and three additional samples after the next dose), most of the applications of this method have been based on obtaining only two samples (peak and trough) from the patient. The following equation is then used for estimating $k$ :

$$
k=\frac{\ln \left(\frac{\text { Peak }}{\text { Trough }}\right)}{\Delta t}
$$

where $\Delta t$ is the difference between the time of peak ( $\left.t_{\text {peak }}\right)$ and time of trough $\left(t_{\text {trough }}\right)$ samples $\left(t_{\text {trough }}-t_{\text {peak }}\right)$, assuming they belong to the same interval. If the samples are taken after the first dose, the peak and trough samples must belong to the same interval. Therefore, there is no confusion about the estimation of $k$ using Equation (1). Additionally, if the peak and trough samples are taken at steady state from the same dosage interval, the use of the above equation is clear. The confusion arises, however, when the peak and trough belong to two different dosage intervals at steady state. This situation arises when, for the sake of convenience and/or expediency, a trough sample is taken first at some interval at steady state, followed by a peak sample collected after the administration of the next dose (Fig. 2). The idea is that at steady state, the peak and trough samples for repeating dosage intervals are theoretically identical. Therefore, the trough sample taken before the next dose, when the peak sample is taken, should be similar to the trough sample if it had been taken at the end of the next dose. Obviously, the use of the actual times written in the patient's chart for application to Equation (1) would yield an incorrect 
$k$ value with a negative sign $\left(t_{\text {trough }}-t_{\text {peak }}\right.$ would be negative) because the slope of the increasing line (from trough to peak) is positive. Therefore, some manipulation of time is needed for estimation of $k$ using Equation (1) and the peak and trough samples from two different intervals: either the clock time of the trough sample should be extended by one dosage interval or the clock time of the peak sample moved back by one dosage interval (Fig. 2). This issue is demonstrated using an example listed below.

\section{Example}

Gentamicin was administered to a patient by a short 0.5 -h infusion of $80 \mathrm{mg}$ every $8 \mathrm{~h}$ starting on 4/26/11. Excerpts from the patient's chart regarding the drug administration and laboratory results for peak and trough concentrations of gentamicin are reported in Fig. 3. As demonstrated in the chart data (Fig. 3) and graphical presentation of the laboratory report (Fig. 2), the trough sample is taken $0.5 \mathrm{~h}$ before the 0930 dose on $4 / 28 / 11$, whereas the peak sample is taken $1.0 \mathrm{~h}$ after the 0930 dose at 1030 . Therefore, the reported peak and trough concentrations belong to two different dosage intervals. Ignoring this fact results in the following inaccurate estimation of $k$ :

$$
k=\frac{\ln \left(\frac{\text { Peak }}{\text { Trough }}\right)}{\Delta t}=\frac{\ln \left(\frac{4.68}{1.37}\right)}{9-10.5}=-0.819 \mathrm{~h}^{-1}
$$

A potential mistake by novice clinicians is to use $(10.5-9)$ in the denominator to obtain a positive value for $k$ (in this case $0.819 \mathrm{~h}^{-1}$ ). Whereas a $\mathrm{k}$ value of $-0.819 \mathrm{hr}^{-1}$ represents an increase in the plasma concentration from trough to peak, a value of $0.819 \mathrm{hr}^{-1}$ represents a decrease in the concentration from peak to trough in $1.5 \mathrm{~h}$. As stated above, in such cases, an accurate estimation of $k$ requires either addition of one dosage interval to the trough time or subtraction of one dosage interval from the peak time, as demonstrated in Figs. 2 and 3. 
Consequently, Equation (1) along with the manipulated times may be used for the accurate estimation of $k$ :

$$
k=\frac{\ln \left(\frac{\text { Peak }}{\text { Trough }}\right)}{\Delta t}=\frac{\ln \left(\frac{4.68}{1.37}\right)}{17-10.5}=0.189 \mathrm{~h}^{-1}
$$

or

$$
k=\frac{\ln \left(\frac{\text { Peak }}{\text { Trough }}\right)}{\Delta t}=\frac{\ln \left(\frac{4.68}{1.37}\right)}{9-2.5}=0.189 \mathrm{~h}^{-1}
$$

A third approach would be to calculate the time within interval for the peak $\left(t_{\text {peak }}\right)$ and trough $\left(t_{\text {trough }}\right)$ samples independently. For this calculation, $t_{\text {peak }}$ is $1 \mathrm{~h}$ because the peak sample was taken $1 \mathrm{~h}$ after the 0930 dose at $1030(1030-0930)$ (Fig. 3). Additionally, $\mathrm{t}_{\text {trough }}$ is $7.5 \mathrm{~h}$ because it was taken $7.5 \mathrm{~h}$ after the 0130 dose at 0900 (0900 - 0130) (Fig. 3). Subsequently, $\Delta \mathrm{t}$ in Equation (1) $\left(\mathrm{t}_{\text {trough }}-\mathrm{t}_{\text {peak }}\right)$ becomes equal to $6.5 \mathrm{~h}(7.5-1.0)$.

\section{Estimation of Volume of Distribution: Based on $C_{\min }$ and $C_{\max }$ or Peak and Trough?}

Estimation of volume of distribution (V) after multiple constant iv infusion is not as simple as that after the iv bolus administration. In their original article [6], Sawchuk and Zaske used the following equation for determination of $\mathrm{V}$ after multiple iv infusions:

$$
V=\frac{R_{o}}{k} \times \frac{\left(1-e^{-k t_{\text {inf }}}\right)}{\left(C_{\text {max }}-C_{\text {predose }} e^{-t_{\text {inf }}}\right)}
$$

where $\mathrm{R}_{\mathrm{o}}, \mathrm{t}_{\text {inf }}, \mathrm{C}_{\max }$, and $\mathrm{C}_{\text {predose }}$ are the rate of short infusion, duration of short infusion, maximum plasma concentration, and the plasma concentration before the administration of the iv

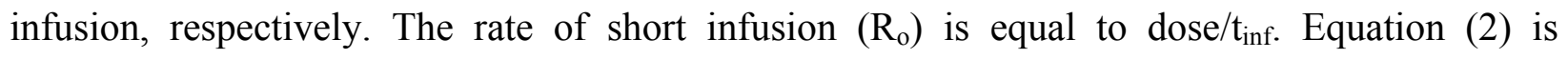
applicable to any dosage interval, including those after the first dose and at steady state. 
Realizing that the $\mathrm{C}_{\text {predose }}$ for the first dose is equal to zero, Equation (2) is simplified after the first dose:

$$
V=\frac{R_{o}}{k} \times \frac{\left(1-e^{\left.-k t_{\text {inf }}\right)}\right.}{C_{\max }}
$$

Additionally at steady state, $\mathrm{C}_{\text {predose }}$ is the same as the steady-state minimum concentration $\left(C_{\min }^{\infty}\right)$, and $\mathrm{C}_{\max }$ is equal to $C_{\max }^{\infty}$ :

$$
V=\frac{R_{o}}{k} \times \frac{\left(1-e^{-k t_{\mathrm{inf}}}\right)}{\left(C_{\max }^{\infty}-C_{\min }^{\infty} e^{-k t_{i n f}}\right)}
$$

Equation (4) may be rearranged to contain only $C_{\max }^{\infty}$ in the denominator, instead of both $C_{\max }^{\infty}$ and $C_{\min }^{\infty}$ :

$$
V=\frac{R_{O}}{k} \times \frac{\left(1-e^{-k t_{\mathrm{inf}}}\right)}{C_{\max }^{\infty}\left(1-e^{-k \tau}\right)}
$$

Both Equations (4) and (5) would give an accurate and identical estimate of V using steady state data. However, in practice, rarely $C_{\max }^{\infty}$ is directly sampled and measured. Instead, a peak sample is taken between 15-60 min after the end of infusion. Therefore, the peak has to be back extrapolated to obtain $C_{\max }^{\infty}$ (Fig. 1) using the following equation:

$$
C_{\max }^{\infty}=\frac{P e a k}{e^{-k \Delta t}}
$$

where $\Delta \mathrm{t}$ is equal to the difference in time between the occurrence of $C_{\max }^{\infty}$ (at the end of infusion) and the peak sampling time. For the example above, $C_{\max }^{\infty}$ is $5.14 \mathrm{mg} / \mathrm{L}$, as demonstrated below:

$$
C_{\max }^{\infty}=\frac{4.68}{e^{-0.189 \times 0.5}}=5.14 \mathrm{mg} / \mathrm{L}
$$

Additionally, the $C_{\min }^{\infty}$ may be obtained from the following equation if the trough sample is taken at a time before the end of interval: 


$$
C_{\text {min }}^{\infty}=\operatorname{Trough} \times e^{-k \Delta t}
$$

where $\Delta t$ in Equation (7) is the time difference between the occurrence of $C_{\min }^{\infty}$ at the end of interval and trough sampling time. In the above example, $C_{\min }^{\infty}$ is equal to $1.25 \mathrm{mg} / \mathrm{L}$ :

$$
C_{\min }^{\infty}=1.37 \times e^{-0.189 \times 0.5}=1.25 \mathrm{mg} / \mathrm{L}
$$

Using the above values in Equation (4), the V for our example case is $19.0 \mathrm{~L}$ :

$$
V=\frac{160}{0.189} \times \frac{\left(1-e^{-0.189 \times 0.5}\right)}{\left(5.14-1.25 \times e^{-0.189 \times 0.5}\right)}=19.0 \mathrm{~L}
$$

Additionally, an identical value of $\mathrm{V}$ may be obtained using Equation (5):

$$
V=\frac{160}{0.189} \times \frac{\left(1-e^{-0.189 \times 0.5}\right)}{5.14 \times\left(1-e^{-0.189 \times 8}\right)}=19.0 \mathrm{~L}
$$

However, some clinicians may use the actual peak and/or trough values, instead of extrapolated $C_{\max }^{\infty}$ and $C_{\min }^{\infty}$, for estimation of patient-specific $\mathrm{V}$. If one uses this approach in the example above, the calculated V will be $22.2 \mathrm{~L}$ using Equation (4):

$$
V=\frac{160}{0.189} \times \frac{\left(1-e^{-0.189 \times 0.5}\right)}{\left(4.68-1.37 \times e^{-0.189 \times 0.5}\right)}=22.2 \mathrm{~L}
$$

Additionally, using peak concentration in Equation (5), instead of $C_{\max }^{\infty}$, results in a V of $20.9 \mathrm{~L}$ :

$$
V=\frac{160}{0.189} \times \frac{\left(1-e^{-0.189 \times 0.5}\right)}{4.68 \times\left(1-e^{-0.189 \times 8}\right)}=20.9 \mathrm{~L}
$$

Using the peak and trough values, instead of $C_{\max }^{\infty}$ and $C_{\min }^{\infty}$, in Equation (4) and using peak value, instead of $C_{\max }^{\infty}$, in Equation (5) resulted in $17 \%$ and $10 \%$ overestimation of $\mathrm{V}$, respectively. Obviously, the magnitude of overestimation will be dependent on the differences in time between the peak and $C_{\max }^{\infty}$ and between the trough and $C_{\min }^{\infty}$, in addition to the magnitude of $k$ : the larger the time difference and/or the $k$, the larger is the error in overestimation of $\mathrm{V}$.

The degree of overestimation of $\mathrm{V}$ using peak concentration, instead of $C_{\max }^{\infty}$, in Equation (5) is demonstrated in Fig. 4 as a function of half life for $\Delta t$ (difference in time between the end 
of infusion and peak) values of $0.25,0.5$, and $1.0 \mathrm{~h}$. As expected, the degree of error is highest for short half lives (large $k$ values) and long $\Delta \mathrm{t}$ values. These simulations (Fig. 4) indicate that when $\Delta \mathrm{t}$ is $0.25 \mathrm{~h}$, the degree of error is less than $10 \%$ as long as the half life is $\geq 2 \mathrm{~h}$, which is the case for most patients. Therefore, using peak value, instead of $C_{\max }^{\infty}$, when $\Delta \mathrm{t}$ is $0.25 \mathrm{~h}$ is not expected to create a sizeable error in most cases. However, for $\Delta \mathrm{t}$ values of 0.5 and $1.0 \mathrm{~h}$, errors greater than $10 \%$ may occur even with short half lives of $\sim 2-3 \mathrm{~h}$. Therefore, it may be prudent to use the extrapolated $C_{\max }^{\infty}$ when $\Delta \mathrm{t}$ is $\geq 0.5 \mathrm{~h}$.

\section{Estimation of Volume of Distribution: Assuming Intravenous Bolus Dosing}

Another approximation in the estimation of $\mathrm{V}$ after multiple iv infusions is to assume the data are obtained after the iv bolus (instead of short infusion) dosing, hence using the following equation:

$$
V=\frac{\text { Dose }}{C_{\text {max }}-C_{\text {predose }}}
$$

where $\mathrm{C}_{\max }$ and $\mathrm{C}_{\text {predose }}$ are defined before. The above equation may be rewritten for the first dose and at steady state:

$$
\begin{gathered}
V=\frac{\text { Dose }}{C_{\max }} \quad \text { First Dose } \\
V=\frac{\text { Dose }}{C_{\max }^{\infty}-C_{\min }^{\infty}} \quad \text { Steady State }
\end{gathered}
$$

Application of this approximation to the above example would result in a V of 20.6 L:

$$
V=\frac{80}{5.14-1.25}=20.6 \mathrm{~L}
$$

Again, this approximation always causes overestimation of $\mathrm{V}$ (in this case by $\sim 8 \%$ ). The degree of overestimation of $\mathrm{V}$ using the equations for bolus route is dependent on the magnitude of difference between $\mathrm{C}_{\mathrm{o}}$ (maximum plasma concentration if the drug were administered by iv 
bolus route) and $\mathrm{C}_{\max }$. This means the longer the length of the infusion or the faster the decline in the plasma concentration (shorter half life), the larger is the difference between the $\mathrm{C}_{\mathrm{o}}$ and $\mathrm{C}_{\max }$ values, hence resulting in a higher overestimation of $\mathrm{V}$.

The degree of overestimation of V using Equation (10), instead of Equation (4) or (5), is demonstrated in Fig. 5 for infusion times of 0.5 and $1.0 \mathrm{~h}$ and dosage intervals of 4, 6, and $8 \mathrm{~h}$. These simulations indicate that in addition to the half life and $t_{\text {inf }}$, the degree of overestimation is also dependent on the dosage interval: Given the same $t_{\text {inf }}$ and $k$, the degree of error is more substantial for shorter dosage intervals (Fig. 5). Additionally, these simulations indicate that when $t_{\text {inf }}$ is equal to $1 \mathrm{~h}$, the degree of overestimation using Equation (10) is high even when the half life is relatively long. Therefore, the use of Equation (10) for estimation of V does not seem appropriate for infusion times of $\geq 1 \mathrm{~h}$.

\section{Estimation of Volume of Distribution: Based on Modified Sawchuk-Zaske Equations}

In addition to the above approximations (using peak and trough values in the SawchukZaske method or using iv bolus equations), some clinicians may use different modifications of the Sawchuk-Zaske method. For example, at Texas Tech School of Pharmacy, some faculty clinicians advocate the use of the following equation:

$$
V=\frac{R_{o}}{k} \times \frac{\left(1-e^{\left.-k t_{\text {inf }}\right)}\right.}{\left(C_{\max }^{\infty}-C_{\min }^{\infty}\right)}
$$

The above equation is different from the exact Sawchuk-Zaske equation by using $C_{\min }^{\infty}$ instead of $C_{\min }^{\infty} e^{-k t_{\text {inf }}}$ in the denominator. Using the above equation for the example data $\left(\mathrm{C}_{\max }\right.$ of 5.14 and $\mathrm{C}_{\min }$ of $1.25 \mathrm{mg} / \mathrm{L}$ ) would yield some degree of overestimation:

$$
V=\frac{160}{0.189} \times \frac{\left(1-e^{-0.189 \times 0.5}\right)}{(5.14-1.25)}=19.6 \mathrm{~L}
$$


Again, the degree of overestimation is dependent on both the elimination rate constant and the length of infusion, in addition to the length of dosage interval. However, in contrast to the approximations based on Equation (10) (Fig. 5), an increase in the half life causes an increase in the degree of overestimation using Equation (11) approximation (Fig. 6). This is because an increase in the half life, keeping $\tau$ constant, also causes an increase in the degree of accumulation of the drug, making the effect of approximation more prominent. Still, similar to Equation (10) approximation, the overestimation is larger for the $t_{\text {inf }}$ of $1 \mathrm{~h}$, compared with that for the $t_{\text {inf }}$ of 0.5 h (Fig. 6). Additionally, the shorter intervals cause a higher degree of error (Fig. 6). Nevertheless, this type of approximation appears to introduce little error for the $t_{\text {inf }}$ of $0.5 \mathrm{~h}$, especially if $\tau$ is $\geq 6 \mathrm{~h}$ (Fig. 6). However, when the $t_{\text {inf }}$ is $1 \mathrm{~h}$, substantial errors may be encountered, depending on the half life and $\tau$ (Fig. 6).

\section{Estimation of Clearance}

Once the $k$ and $\mathrm{V}$ values are estimated above, the clearance (Cl) of aminoglycosides may be simply obtained using the following equation:

$$
C l=k \times V
$$

The degree of error in the estimation of $\mathrm{Cl}$ is obviously dependent on the errors associated with the estimation of $k$ and $\mathrm{V}$ as discussed above.

\section{DESIGN OR ADJUSTMENT OF DOSAGE REIMENS BASED ON THE SAWCHUK- ZASKE EQUATIONS}

The Sawchuk-Zaske equation for the determination of $\mathrm{V}$ at steady-state may be rearranged to estimate the infusion rate $\left(\mathrm{dose} / \mathrm{t}_{\mathrm{inf}}\right)$ necessary to achieve desired maximum and 
minimum concentrations. Additionally, the desired maximum and minimum concentrations and the drug elimination rate constant may be used to estimate an appropriate dosage interval. To design a dosage regimen (dosage interval and infusion rate), one may use the initial kinetic parameters obtained based on the population data or the patient-specific parameters. Estimations of dosage interval and infusion rate are explained in more detail below.

\section{Estimation of Dosage Interval: Based on Desired $C_{\min }$ and $C_{\max }$ or Peak and Trough?}

One of the sources of confusion in the literature regarding aminoglycoside dosing is that the goal of therapy in most cases is stated based on peak and trough values [1], rather than $C_{\max }^{\infty}$ and $C_{m i n}^{\infty}$. Therefore, in designing the dosage regimens, it must be decided whether the goal is to achieve a certain $C_{\max }^{\infty}$ and $C_{\min }^{\infty}$ or a certain peak and trough. Obviously, if the goal is stated in terms of peak and trough, it must be defined in terms of the distances between the peak and $C_{\max }^{\infty}$ and between the trough and $C_{\min }^{\infty}$. The following equation may be used to estimate a $\tau$ based on desired maximum and minimum concentrations:

$$
\tau=\frac{\ln \left(\frac{C_{\max }^{\infty}}{C_{\min }^{\infty}}\right)}{k}+t_{\text {inf }}
$$

For example, in the above case, if the goal is to achieve a $C_{\max }^{\infty}$ of $8 \mathrm{mg} / \mathrm{L}$ and a $C_{\min }^{\infty}$ of $\leq 2$ $\mathrm{mg} / \mathrm{L}$, the calculated $\tau$ will be $7.83 \mathrm{~h}$ :

$$
\tau=\frac{\ln \left(\frac{8}{2}\right)}{0.189}+0.5=7.83 \mathrm{~h}
$$

One needs to round the calculated $\tau$ to a practical $\tau$, in this case $8 \mathrm{~h}$. However, if the goal is expressed as desired trough and peak concentration, the following equation may be used:

$$
\tau=\frac{\ln \left(\frac{\text { Peak }}{\text { Trough }}\right)}{k}+t_{\text {inf }}+\Delta t_{\text {peak }}+\Delta t_{\text {trough }}
$$


where $\Delta t_{\text {peak }}$ and $\Delta t_{\text {trough }}$ represent, respectively, the difference in time between the peak and maximum concentration and between the trough and minimum concentration at steady-state. If we assume that the desired peak and trough are based on a situation when peak is taken $0.5 \mathrm{~h}$ after the end of infusion and trough is taken immediately before the next dose, the $\Delta t_{\text {peak }}$ and $\Delta t_{\text {trough }}$ values will be $0.5 \mathrm{~h}$ and zero, respectively:

$$
\tau=\frac{\ln \left(\frac{8}{2}\right)}{0.189}+0.5+0.5+0.0=8.33 \mathrm{~h}
$$

The calculated $\tau$ in this case is more than $8 \mathrm{~h}$. Rounding down the calculated $\tau$ to $8 \mathrm{~h}$ means that the expected trough value using this regimen will be higher than $2 \mathrm{mg} / \mathrm{L}$.

\section{Estimation of Infusion Rate: Based on Desired $\mathrm{C}_{\max }$ or Peak?}

Similar to the estimation of $\tau$ above, the estimation of infusion rate $\left(\mathrm{R}_{\mathrm{o}}\right.$ or dose $\left./ \mathrm{t}_{\mathrm{inf}}\right)$ is also dependent on the desired therapeutic goal. If the goal is based on the desired $C_{\max }^{\infty}$, then the following equation is used:

$$
\begin{gathered}
R_{o}=C_{\text {max }}^{\infty} \times V \times k \times \frac{\left(1-e^{-k \tau}\right)}{\left(1-e^{-k t_{\text {inf }}}\right)} \\
R_{o}=8 \times 19 \times 0.189 \times \frac{\left(1-e^{-0.189 \times 8}\right)}{\left(1-e^{-0.189 \times 0.5}\right)}=254 \mathrm{mg} / \mathrm{h}
\end{gathered}
$$

Based on the above calculations, the new dose (dose $\left.=\mathrm{R}_{\mathrm{o}} \mathrm{x} \mathrm{t}_{\text {inf }}\right)$ to achieve a $C_{\max }^{\infty}$ of $8 \mathrm{mg} / \mathrm{L}$ is $\sim 130 \mathrm{mg}($ dose $=254 \times 0.5)$, administered over a $0.5 \mathrm{~h}$ short infusion. On the other hand, if the goal is to achieve a peak of $8 \mathrm{mg} / \mathrm{L}$ at $0.5 \mathrm{~h}$ after the end of infusion, one may use the following equation:

$$
\begin{gathered}
R_{o}=\frac{P e a k}{e^{-k \Delta t}} \times V \times k \times \frac{\left(1-e^{-k \tau}\right)}{\left(1-e^{-k t_{\mathrm{inf}}}\right)} \\
R_{o}=\frac{8}{e^{-0.189 \times 0.5}} \times 19 \times 0.189 \times \frac{\left(1-e^{-0.189 \times 8}\right)}{\left(1-e^{-0.189 \times 0.5}\right)}=280 \mathrm{mg} / \mathrm{h}
\end{gathered}
$$


This means a dose of $140 \mathrm{mg}$ administered over a $0.5 \mathrm{~h}$ short infusion. Obviously, a peak of 8 $\mathrm{mg} / \mathrm{L}$ in this case means a $C_{\max }^{\infty}$ of $8.8 \mathrm{mg} / \mathrm{L}$ :

$$
C_{\max }^{\infty}=\frac{\text { Peak }}{e^{-k \Delta t}}=\frac{8}{e^{-0.189 \times 0.5}}=8.8 \mathrm{mg} / \mathrm{L}
$$

The difference in $\mathrm{R}_{\mathrm{o}}$ (or dose) between the two methods (using $C_{\max }^{\infty}$ or peak) is dependent on the magnitude of $e^{-k \Delta t}$, where $\Delta \mathrm{t}$ is the difference between the time of peak sampling and end of infusion.

\section{OTHER SOURCES OF ERROR}

In addition to the above errors associated with the use of modified versions of the Sawchuk-Zaske method, there are other potential sources of error that are not unique to this method of calculation. For example, inaccuracies in recording the exact time of sampling or dosing could potentially result in significant errors in the final kinetic parameters and the dose. Further, in some cases, $R_{0}$, which is dose/ $t_{\text {inf }}$, may be mistaken with the dose. The latter is especially troublesome if $t_{\text {inf }}$ is $0.5 \mathrm{~h}$, resulting in administration of a dose that is twice as much as the intended dose. Therefore, some protocols advocate the use of $1 \mathrm{~h}$ infusions to avoid this potential problem.

\section{CONCLUSIONS AND RECOMMENDATIONS}

Because in clinical settings, the peak and trough samples for aminoglycosides are usually taken from two consecutive intervals at steady state, it is recommended that clinical pharmacology curricula expose novice clinicians to this type of calculation during their education. Further, as simulations in this communication revealed, the degree of error associated 
with the estimation of aminoglycosides $\mathrm{V}$ using modified forms of the Sawchuk-Zaske or iv bolus equations, instead of the original equations, may be significant. Although the experts may use modified equations, understanding their error ramifications, these modifications may be confusing to the novice clinicians. Therefore, it is recommended that Equation (4) or (5) be used without any modification or simplification in calculation of $\mathrm{V}$. As for the design of new dosage regimens, it has to be decided first whether the goal of treatment is to achieve certain $C_{\max }^{\infty}$ and $C_{\min }^{\infty}$ or certain trough and peak. If the former is selected, Equations (13) and (15) should be used for the determination of the dosage interval and infusion rate, respectively. If the latter is selected, the peak and trough must be defined in terms of their time distance from the $C_{\max }^{\infty}$ and $C_{m i n}^{\infty}$, respectively. In that case, Equations (14) and (16) may be used for the estimation of the dosage interval and infusion rate, respectively.

\section{CONFLICT OF INTEREST}

The author declares no conflict of interest with the content of this manuscript. 


\section{REFERENCES}

[1] Gonzalez LS, 3rd, Spencer JP. Aminoglycosides: a practical review. Am Fam Physician $1998 ; 58: 1811-20$.

[2] Moore RD, Lietman PS, Smith CR. Clinical response to aminoglycoside therapy: importance of the ratio of peak concentration to minimal inhibitory concentration. J Infect Dis 1987 ; $155: 93-9$.

[3] Dahlgren JG, Anderson ET, Hewitt WL. Gentamicin blood levels: a guide to nephrotoxicity. Antimicrob Agents Chemother 1975; 8:58-62.

[4] Xuan D, Lu JF, Nicolau DP, Nightingale CH. Population pharmacokinetics of tobramycin in hospitalized patients receiving once-daily dosing regimen. Int J Antimicrob Agents $2000 ; 15: 185-91$.

[5] Zaske DE, Cipolle RJ, Rotschafer JC, Solem LD, Mosier NR, Strate RG. Gentamicin pharmacokinetics in 1,640 patients: method for control of serum concentrations. Antimicrob Agents Chemother 1982; 21:407-11.

[6] Sawchuk RJ, Zaske DE. Pharmokinetics of dosing regimens which utilize mutiple intravenous infusions: gentamicin in burn patients. J Pharmacokinet Biopharm 1976; 4:183-95.

[7] Kovarik JM, Hoepelman IM, Verhoef J. Once-daily aminoglycoside administration: new strategies for an old drug. Eur J Clin Microbiol Infect Dis 1989; 8:761-9.

[8] Schentag J, Meagher A, Jelliffe R. Aminoglycosides. In: Burton M, Shaw L, Schentag JJ, Evans WE, Eds. Applied Pharmacokinetics \& Pharmacodynamics: Principles of Therapeutic Drug Monitoring. 4th ed. Lippincott Williams \& Wilkins: Philadelphia 2006; pp. 40-59. 
[9] Bauer LA. The aminoglycoside antibiotics. In: Applied Clinical Pharmacokinetics. 2nd ed. McGraw-Hill: New York 2008; pp. 97-206.

[10] Beringer P, Winter ME. Aminoglycoside antibiotics. In: Winter ME, Ed. Basic Clinical Pharmacokinetics. 4th ed. Lippincott Williams \& Wilkins: Philadelphia 2004; pp. 130-71.

[11] Touw DJ, Westerman EM, Sprij AJ. Therapeutic drug monitoring of aminoglycosides in neonates. Clin Pharmacokinet 2009; 48:71-88.

[12] Craig WA. Optimizing aminoglycoside use. Crit Care Clin 2011; 27:107-21.

[13] Mueller EW, Boucher BA. The use of extended-interval aminoglycoside dosing strategies for the treatment of moderate-to-severe infections encountered in critically ill surgical patients. Surg Infect 2009; 10:563-70. 


\section{LEGEND FOR FIGURES}

Fig. (1). The differences in the drug plasma/serum concentration-time profiles between iv bolus dose and short iv infusion methods for a hypothetical case. The same dose is administered in both cases. $\mathrm{C}_{0}$, concentration immediately after the iv bolus dose; $\mathrm{C}_{\max }$, concentration immediately after a short infusion of the same dose; $\mathrm{C}_{\min }$, the minimum concentration before the next dose; Peak, the concentration some time (usually 15-60 min) after the end of infusion; Trough, the concentration some time (usually $\leq 30 \mathrm{~min}$ ) before $\mathrm{C}_{\min }$; $t_{\text {inf }}$, the length of short infusion (usually 30 or $60 \mathrm{~min}$ ); $\tau$, the length of dosage interval.

Fig. (2). Simulated serum/plasma concentration-time profiles of an example drug adminsitered at a dose of $80 \mathrm{mg}$ over a $30 \mathrm{~min}$ short iv infusion (infusion rate of $160 \mathrm{mg} / \mathrm{h}$ ) every $8 \mathrm{~h}$. The drug is administered at 0130, 0930, and 1730 every day with the first dose adminsitered at 0930. A trough and a peak sample were taken at $0.5 \mathrm{~h}$ before and $1.0 \mathrm{~h}$ after the start of the seventh short infusion, respectively (bold circles). Moving the peak backward or the trough forward by one dosage interval is necessary for estimation of rate constant using Equation (1) in the text.

Fig. (3). Excerpts from a patient's chart describing the medication and relevant laboratory results. Additionally, the manipulation of sampling time of trough or peak necessary for accurate estimation of patient-specific elimination rate constant using Equation (1) is also demonstrated.

Fig. (4). Degree of overestimation of volume of distribution as a function of drug half life when peak concentration is used in Equation (5) instead of the maximum concentration. The data are 
simulated for three cases with the differences in time $(\Delta t)$ between the occurrence of maximum concentration (at the end of infusion) and the peak sampling time being $0.25,0.5$, or $1.0 \mathrm{~h}$. The length of infusion does not affect the degree of overestimation. The horizontal line demonstrates an arbitrary error of $10 \%$.

Fig. (5). Degree of overestimation of volume of distribution as a function of drug half life when iv bolus Equation (10) is used instead of multiple iv infusion Equation (4) or (5). The data are simulated for three dosage intervals of 4,6 , and $8 \mathrm{~h}$ and infusion lengths of $0.5 \mathrm{~h}$ (top) and $1.0 \mathrm{~h}$ (bottom). The horizontal line demonstrates an arbitrary error of $10 \%$.

Fig. (6). Degree of overestimation of volume of distribution as a function of drug half life when the approximation Equation (11) is used instead of the exact Equation (4) or (5). The data are simulated for three dosage intervals of 4,6 , and $8 \mathrm{~h}$ and infusion lengths of $0.5 \mathrm{~h}$ (top) and $1.0 \mathrm{~h}$ (bottom). The horizontal line demonstrates an arbitrary error of $10 \%$. 


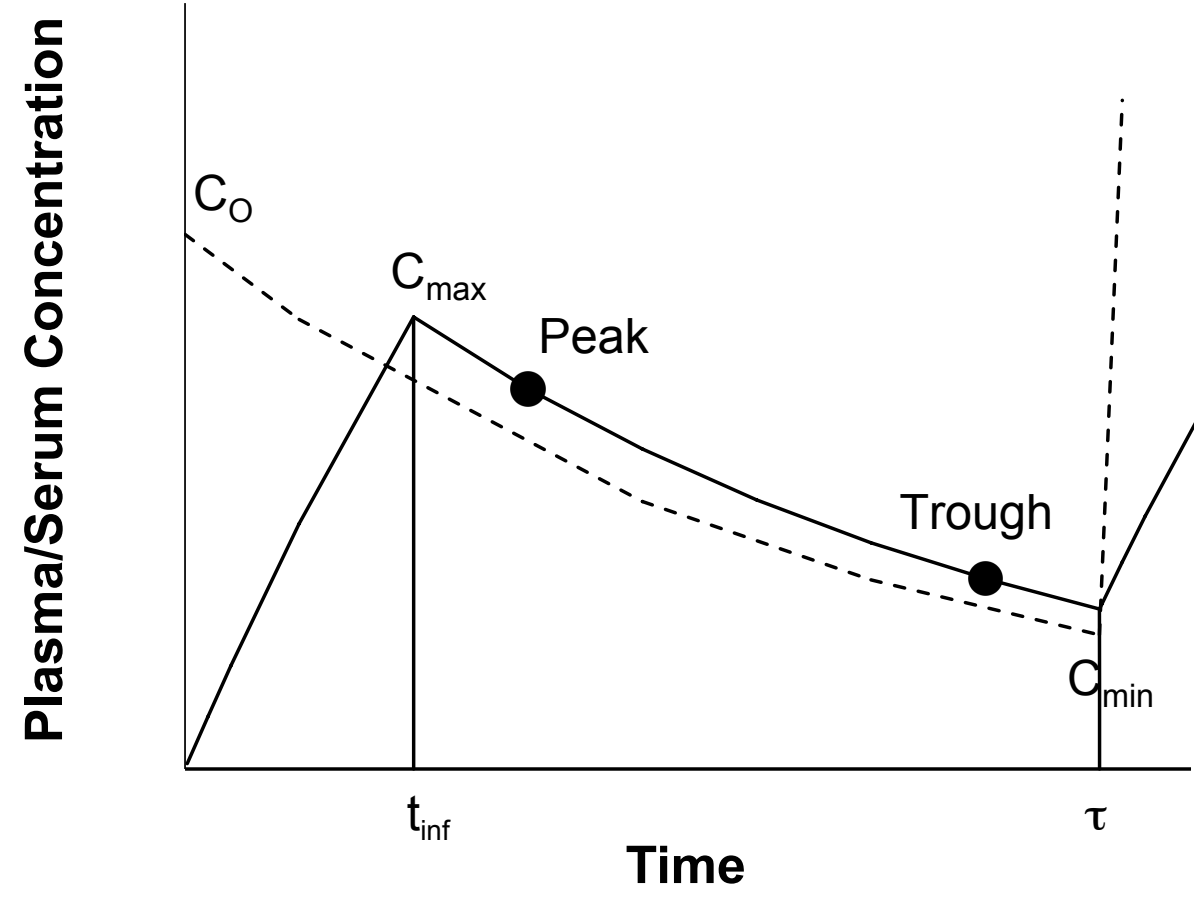

Fig. 1 


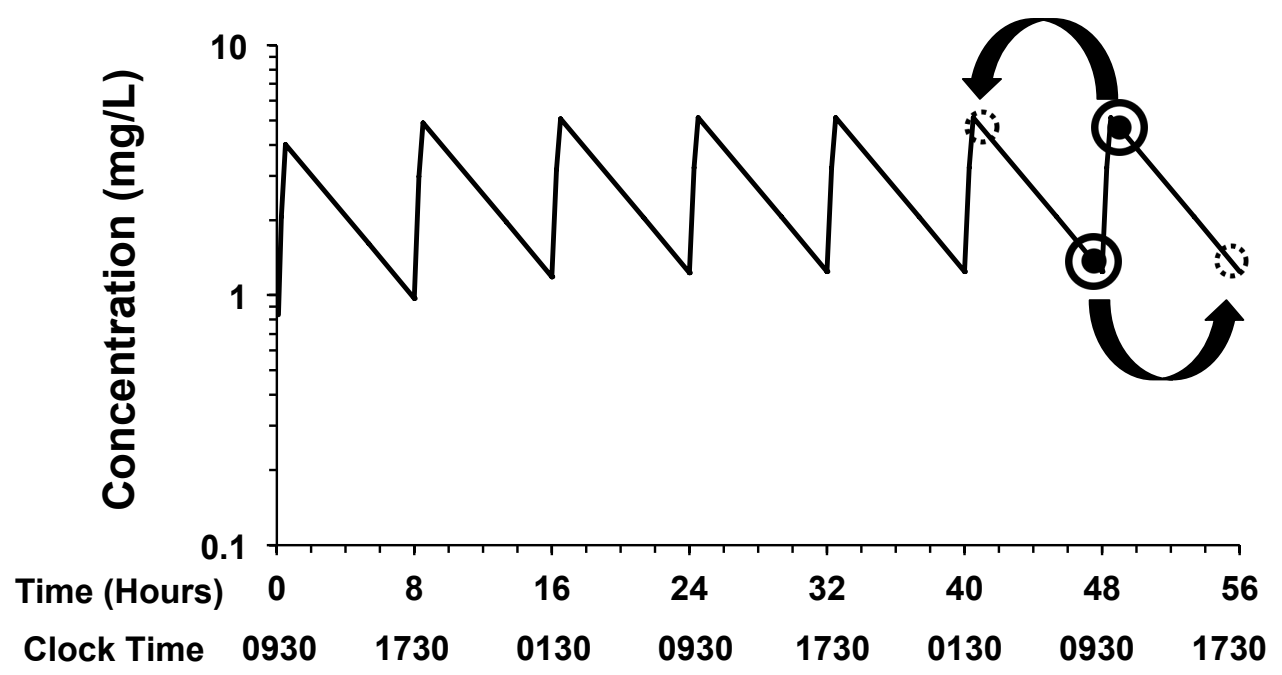

Fig. 2 


\section{Excerpts from Medication Administration Record:}

Gentamicin $80 \mathrm{mg}$ Q8H

IV infusion over $30 \mathrm{~min}$

Start 4/26/11 $0930 \quad 1730 \quad 0130$

Excerpts from Laboratory Report:

\begin{tabular}{|l|l|l|}
\hline \multicolumn{3}{|c|}{ Gentamicin Serum Concentration } \\
\hline Date & $4 / 28 / 11$ & $4 / 28 / 11$ \\
\hline Time & 1030 & 0900 \\
\hline Concentration $(\mathrm{mg} / \mathrm{L})$ & 4.68 & 1.37 \\
\hline
\end{tabular}

Manipulation of Trough or Peak Time

Moving the Trough One Interval Forward

\begin{tabular}{|c|c|c|}
\hline & Peak & Trough \\
\hline Time (hr) & 1030 & 1700 \\
\hline Concentration $(\mathrm{mg} / \mathrm{L})$ & 4.68 & 1.37 \\
\hline \multicolumn{3}{|c|}{ Moving the Peak One Interval Backward } \\
\hline & Peak & Trough \\
\hline Time (hr) & 0230 & 0900 \\
\hline Concentration (mg/L) & 4.68 & 1.37 \\
\hline
\end{tabular}

Fig. 3 


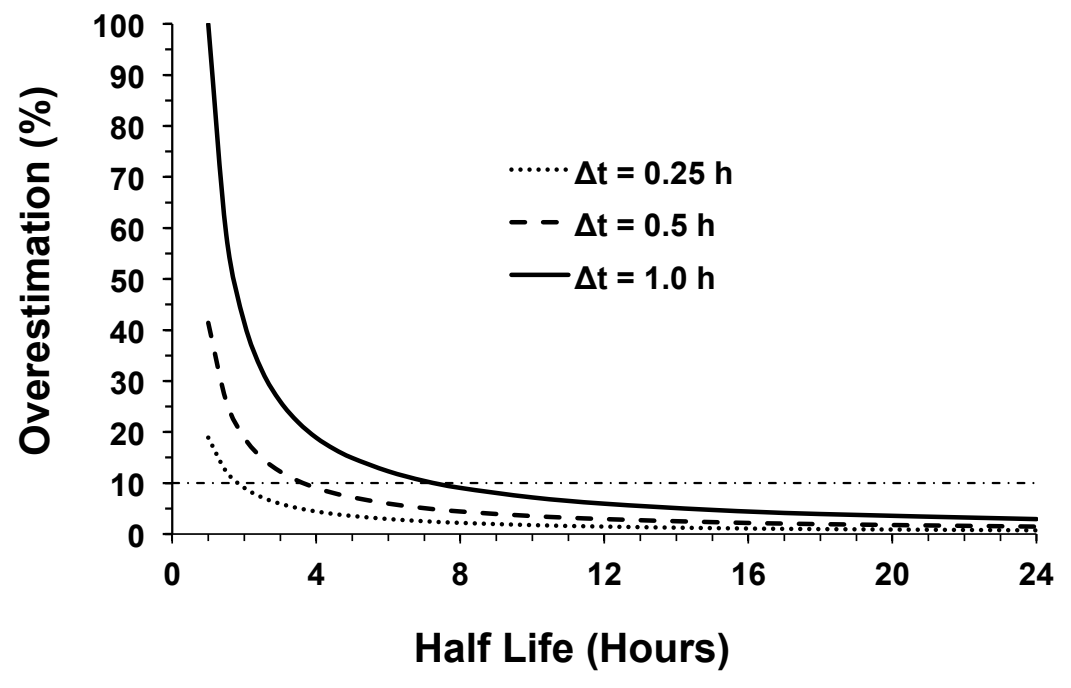

Fig. 4 

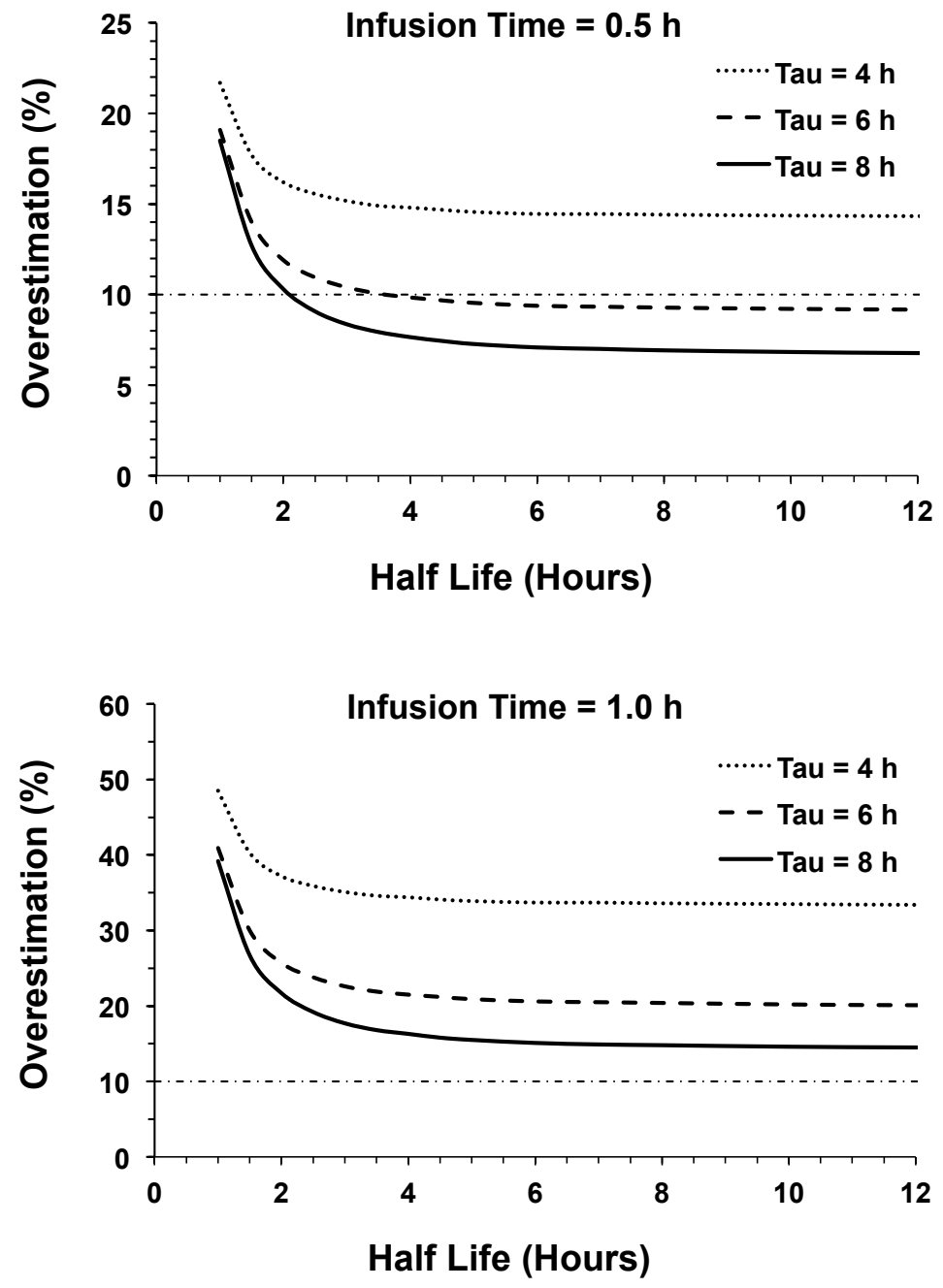

Fig. 5 

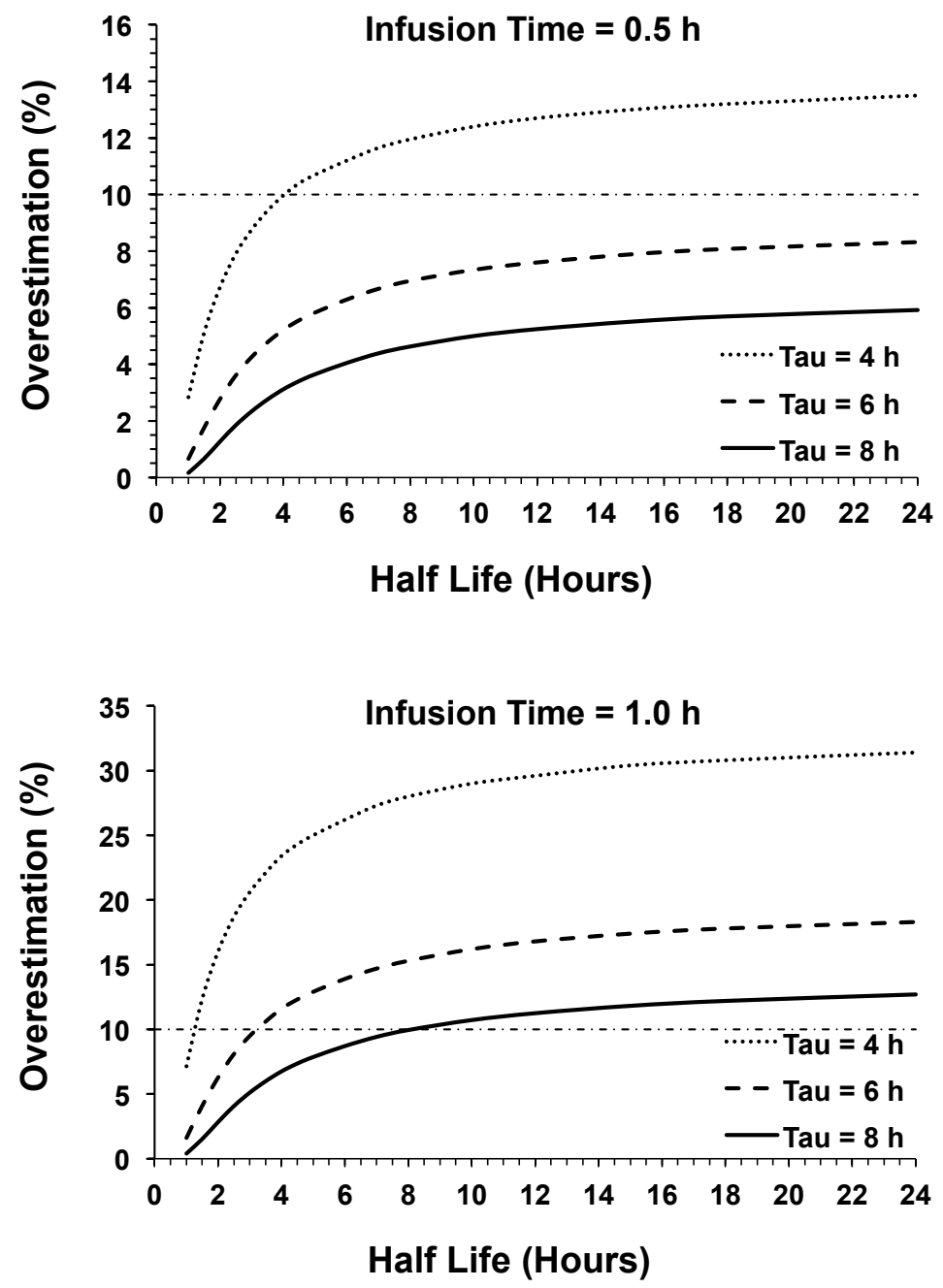

Fig. 6 